\title{
LX. The periodic law, as illustrated by certain physical properties of organic compounds.-Part II. The melting-and boiling-points of the halogen and alkyl compounds of the hydrocarbon radicals
}

\author{
Thomas Carnelley D.Sc.
}

To cite this article: Thomas Carnelley D.Sc. (1885) LX. The periodic law, as illustrated by certain physical properties of organic compounds.-Part II. The melting-and boiling-points of the halogen and alkyl compounds of the hydrocarbon radicals, Philosophical Magazine Series 5, 20:127, 497-514, DOI: 10.1080/14786448508627794

To link to this article: http://dx.doi.org/10.1080/14786448508627794

\section{Published online: 29 Apr 2009.}

Submit your article to this journal ¿

\section{Џ Article views: 2}

Q View related articles ¿ 
TABLE II.-Showing the effect of Length and of Hardness on the Induction-Coefficient of Magnets.

\begin{tabular}{|c|c|c|c|c|c|c|}
\hline \multirow[b]{2}{*}{$\begin{array}{l}\text { Length } \\
\text { of bar in } \\
\text { centi- } \\
\text { metres. }\end{array}$} & \multirow[b]{2}{*}{$\begin{array}{l}\text { Ratio of } \\
\text { length to } \\
\text { diameter. }\end{array}$} & \multicolumn{2}{|c|}{ Unit field. } & \multirow[b]{2}{*}{$\begin{array}{c}\text { Mean of } \\
\text { numbers in } \\
\text { columns } \\
3 \text { and } 4 .\end{array}$} & \multirow[b]{2}{*}{$\begin{array}{c}\text { Magnetic } \\
\text { moment } \\
\text { per } \\
\text { gramme. }\end{array}$} & \multirow[b]{2}{*}{ Remarks. } \\
\hline & & $\begin{array}{c}\text { Apparent } \\
\text { percentage } \\
\text { increase of } \\
\text { moment for } \\
\text { unit field: } \\
\text { side-on } \\
\text { position. }\end{array}$ & $\begin{array}{c}\text { Apparent } \\
\text { percentage } \\
\text { increase of } \\
\text { moment for } \\
\text { unit field: } \\
\text { end-on } \\
\text { position. }\end{array}$ & & & \\
\hline 3 & 10 & 0.80 & 0.90 & $0 \cdot 85$ & 27 & Glass hard \\
\hline 4 & 16 & $0 \cdot 67$ & 0.73 & 0.70 & 32 & $"$ \\
\hline 4 & 16 & 0.67 & $0 \cdot 70$ & $0 \cdot 69$ & 35 & $"$ \\
\hline 6 & 20 & 0.51 & 067 & $0 \cdot 59$ & 36 & $"$ \\
\hline 7 & 31 & 0.51 & 0.58 & 0.54 & 39 & " \\
\hline 8 & 32 & 0.51 & 0.58 & $0 \cdot 54$ & 54 & $"$ \\
\hline 8 & 32 & 0.51 & 0.58 & 0.54 & 52 & ", \\
\hline 10 & 34 & $9 \cdot 46$ & 0.56 & 0.51 & 40 & " \\
\hline 10 & 44 & 0.40 & $0 \cdot 56$ & 0.48 & 43 & $"$ \\
\hline 7 & 47 & $0 \cdot 46$ & $0 \cdot 51$ & $0 \cdot 49$ & 57 & $"$ \\
\hline 10 & 50 & 0.44 & 0.58 & 0.51 & 67 & $"$ \\
\hline 10 & 50 & 0.48 & 0.54 & 0.51 & 60 & $"$ \\
\hline 10 & 50 & $0 \cdot 46$ & 0.55 & 0.51 & 53 & $"$ \\
\hline 10 & 50 & 0.46 & 0.52 & 0.49 & 71 & , \\
\hline 10 & 50 & $0 \cdot 46$ & 0.56 & 0.51 & 60 & $"$ \\
\hline 10 & 67 & 0.41 & 0.51 & $0 \cdot 46$ & 65 & $"$ \\
\hline 7 & 73 & $0 \cdot 41$ & 0.50 & 0.47 & 64 & $"$ \\
\hline 10 & 105 & 0.42 & 0.45 & 0.43 & 66 & ", \\
\hline 10 & 34 & $0 \cdot 47$ & 0.53 & 0.50 & $41 \cdot 5$ & Glass hard. \\
\hline 10 & 34 & $0 \cdot 63$ & 0.67 & $0 \cdot 65$ & $44 \cdot 5$ & Yellow. \\
\hline 10 & 34 & 0.84 & 0.98 & 0.91 & $54 \cdot 1$ & Blue. \\
\hline 10 & 48 & $0 \cdot 32$ & 0.40 & $0 \cdot 36$ & 45 & Glass hard. \\
\hline 10 & 48 & 0.43 & 0.55 & 0.49 & 46 & Yellow. \\
\hline 10 & 48 & 0.53 & 0.67 & 0.60 & 71 & \\
\hline
\end{tabular}

LX. The Periodic Law, as Illustrated by certain Physical Properties of Organic Compounds.-Part II. The Meltingand Boiling-points of the Halogen and Alkyl Compounds of the Hydrocarbon Radicals. By Thomas CaRneliey, D.Sc., Professor of Chemistry in University College, Dundee*.

TN the present series of papers my object is to determine 1 whether the elements are in any way analogous to the hydrocarbon radicals of Organic Chemistry. For this purpose

* Communicated by the Author. 
I have made a careful comparison of the physical properties (chiefly melting- and boiling-points) of a large number of both organic and inorganic compounds, and believe that I have been able to make out numerous and varied relationships between these properties.

My first paper (Phil. Mag. [5] xviii. p. 1) dealt with the melting- and boiling-points and heats of formation of the normal halogen compounds of the elements, and I showed that certain well-defined relationships existed as regards the above physical properties of these compounds. $\mathrm{My}_{\mathrm{y}}$ second paper (ibid. [5] xx. p. 259) dealt in a similar manner with some of the physical properties of the normal alkyl compounds of the elements, which were shown to exhibit exactly the same relationships as those of the corresponding halogen compounds; proving, therefore, that the function of the alkyl radicalsmethyl $\mathrm{CH}_{3}$, ethyl $\mathrm{C}_{2} \mathrm{H}_{5}$, propyl $\mathrm{C}_{3} \mathrm{H}_{7}$, \&c.-was exactly analogous to that of the balogen elements-chlorine, bromine, and iodine.

In the present paper I wish to show that the normal halogen and alkyl compounds of the hydrocarbon radicals exhibit relationships similar to those of the corresponding compounds of the elements, and therefore that the elements and hydrocarbon radicals are analogous and have the same function in their several compounds. In a fourth and final paper $I$ intend to take a general review of the whole question, and to draw certain conclusions as to the nature of the chemical elements and of the Periodic Law.

The following table contains the experimental data, with the authorities, which have been used in drawing the conclusions given below. For the purpose of avoiding minus signs all temperatures are reckoned from the absolute zero $(-273)$. The following abbreviations are used :- $\mathrm{Me}=$ methyl, $\mathrm{CH}_{3}$; $\mathrm{Et}=$ ethyl, $\mathrm{C}_{2} \mathrm{H}_{5} ; \mathrm{Pr}^{\alpha}=$ normal propyl, $\mathrm{CH}_{3} \cdot \mathrm{CH}_{2} \cdot \mathrm{CH}_{2}-$; $\mathrm{Pr}^{\beta}=$ isopropyl $\left(\mathrm{CH}_{3}\right)_{2} \mathrm{CH}-$. 
Prof. T. Carnelley on the Periodic Law.

TABLE I.-Experimental Data.

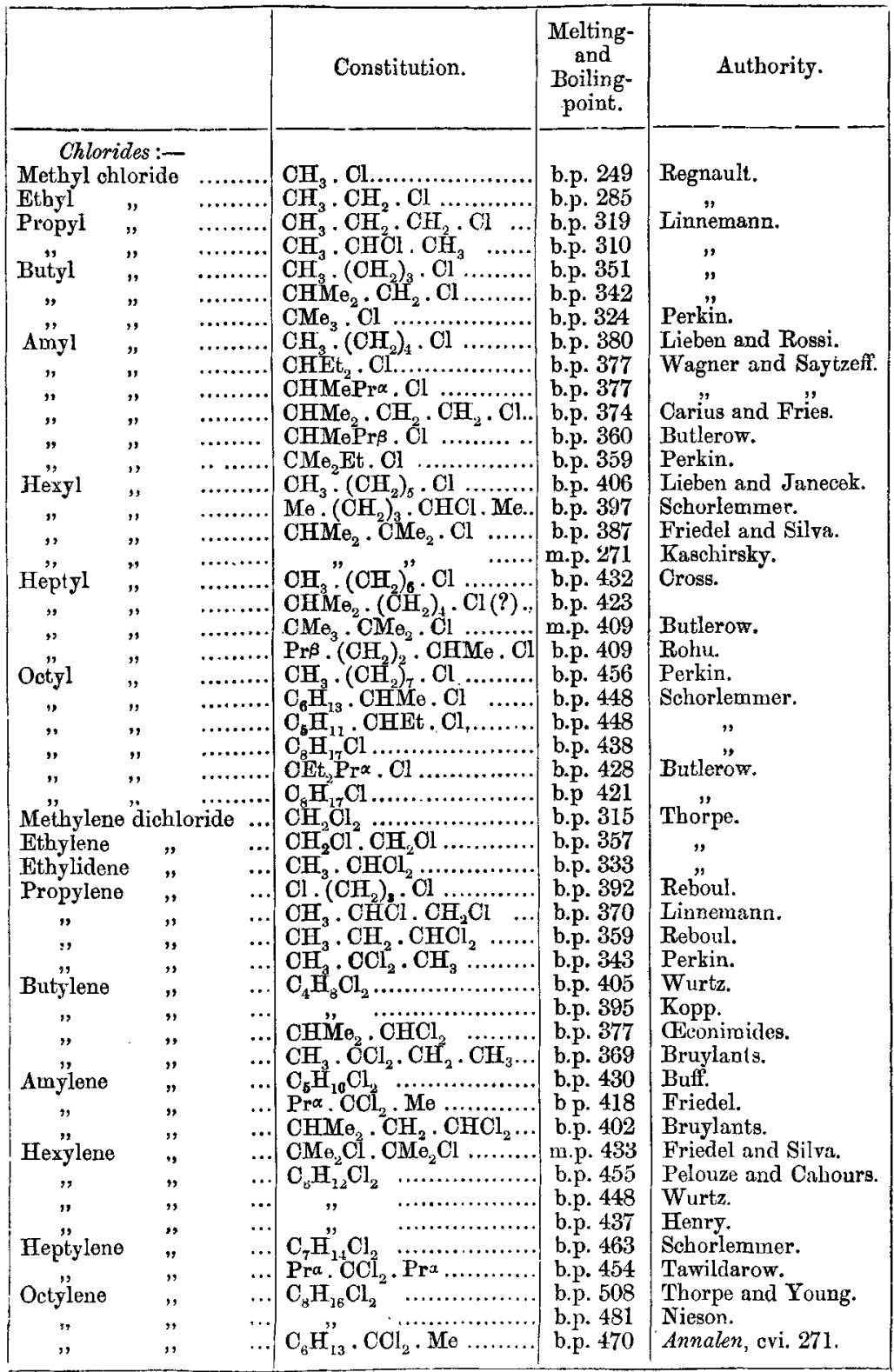


TABLE I. (continued).

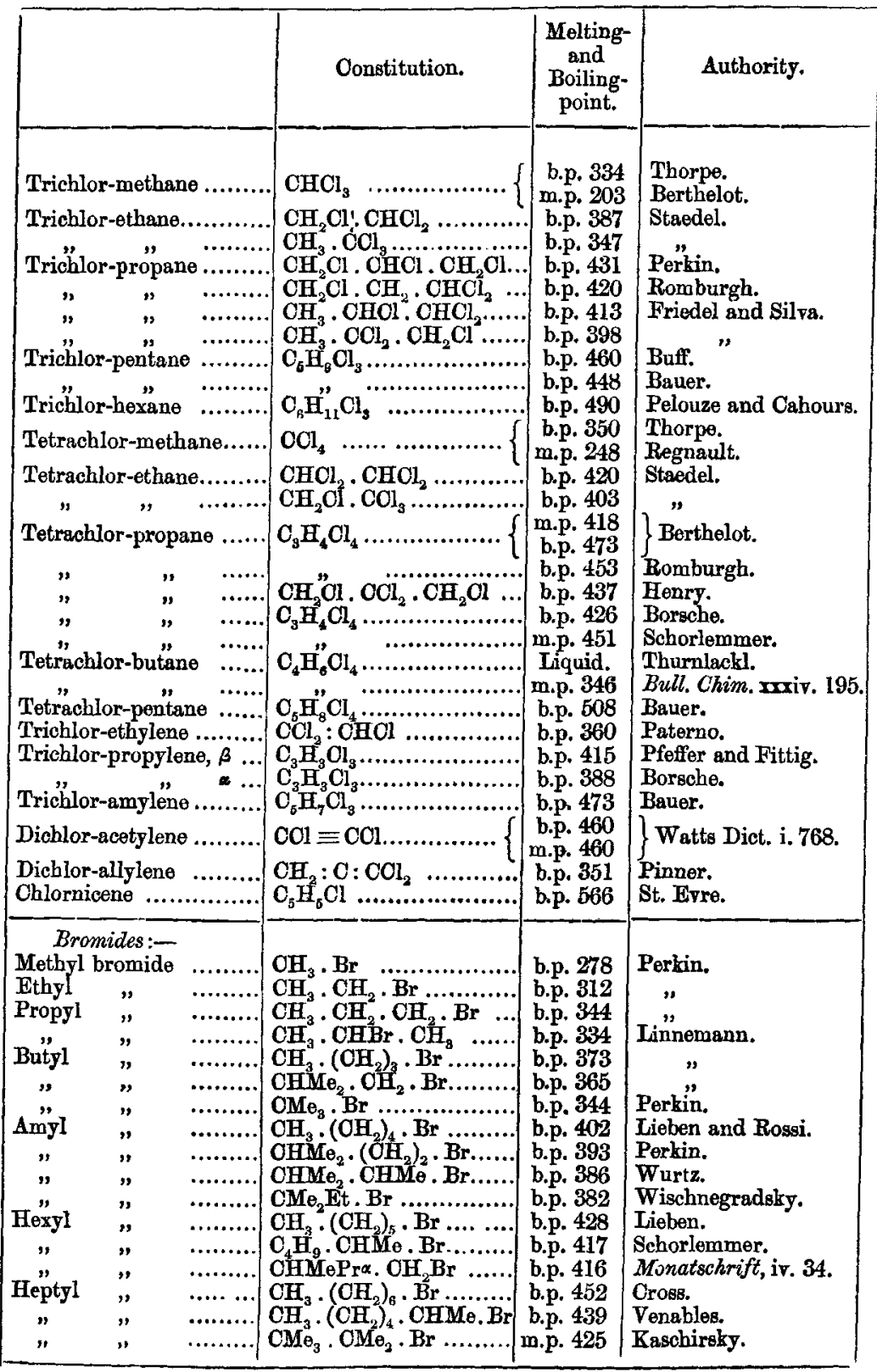


TABLE I. (continued).

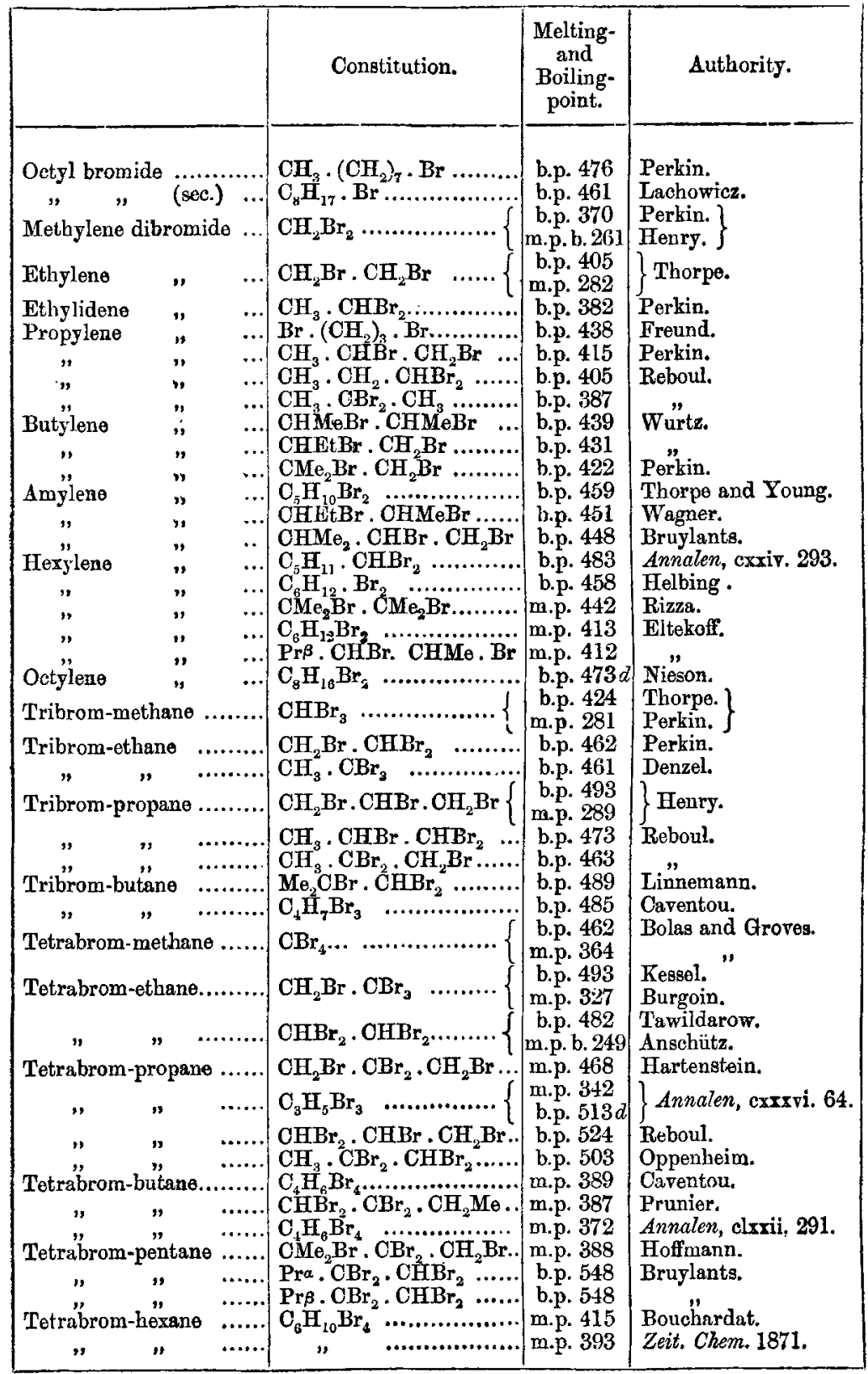

Phil. Mag. S. 5. Vol. 20. No. 127. Dec. 1885. 
TABLE I. (continued).

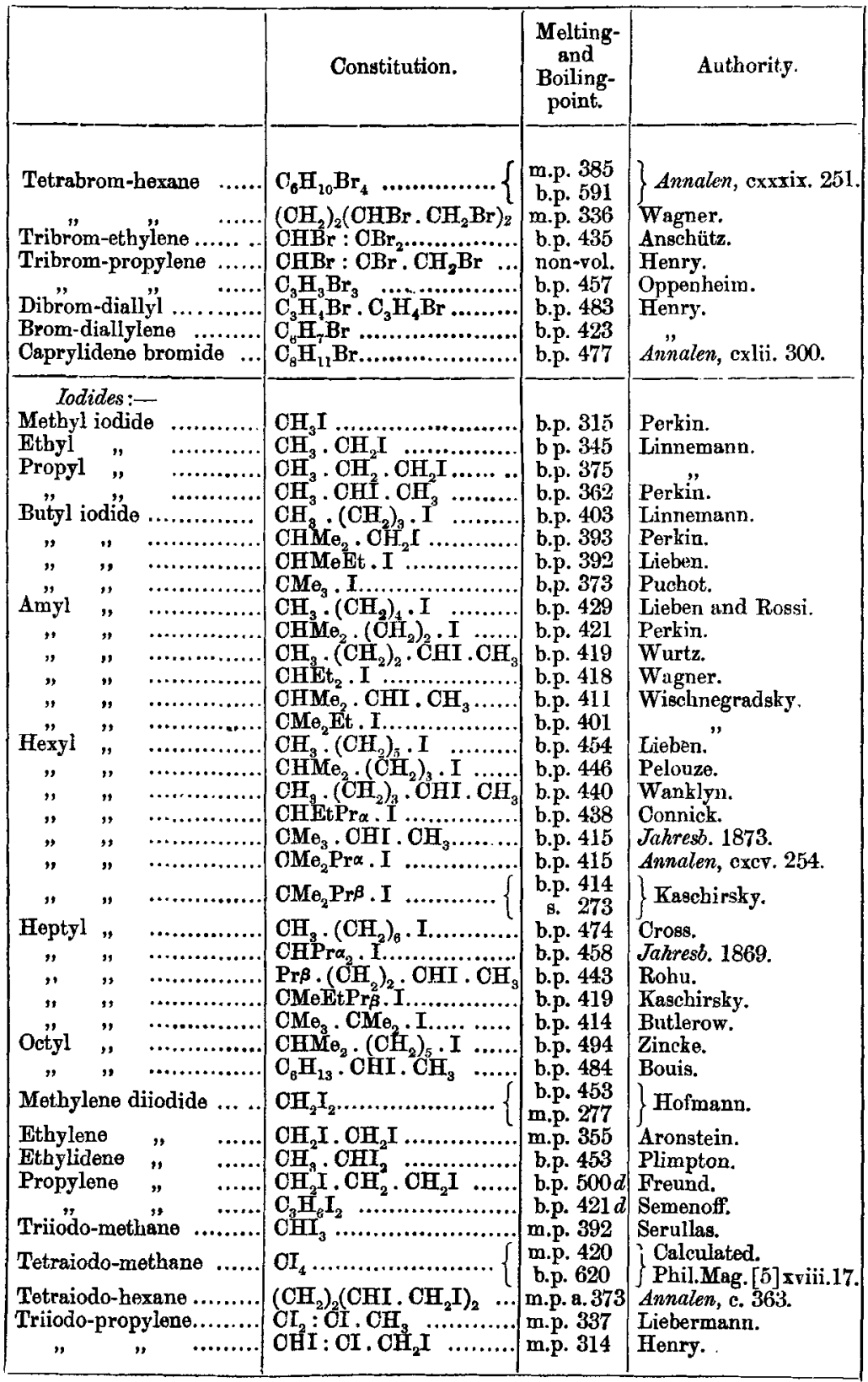


TABLE I. (continued).

\begin{tabular}{|c|c|c|c|}
\hline & Constitution. & $\begin{array}{l}\text { Melting- } \\
\text { and } \\
\text { Boiling- } \\
\text { point. }\end{array}$ & Authority. \\
\hline Alkyl compounds $\left(\mathrm{H}_{\mathrm{y}}\right.$ & drocarbons) :- & & \\
\hline Propane........................ & Normal ..... & b.p. 245 & Lefebrre. \\
\hline $\begin{array}{c}\text { Butane } \\
,, \\
, \ldots \ldots . . . . . . . \\
\end{array}$ & $\mathrm{CHM}_{3} \ldots$ & $\begin{array}{l}\text { b.p. } 274 \\
\text { b.p. } 256\end{array}$ & $\begin{array}{l}\text { Schorlemmer. } \\
\text { Butlerow. }\end{array}$ \\
\hline 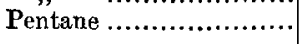 & Normal.. & b.p. 311 & Schorlemmer. \\
\hline ................... & $\mathrm{CHMe}_{2} \cdot \mathrm{OH}_{2} \cdot \mathrm{Me}$ & b.p. 303 & Pelouze. \\
\hline . $\ldots \ldots \ldots \ldots \ldots \ldots$ & $\mathrm{OMe}_{4} \ldots \ldots \ldots \ldots$ & $\begin{array}{l}\text { b.p. } 282 \\
\text { s. } 253\end{array}$ & Lwow. \\
\hline Hexane ............... & Normal ......... & b.p. 344 & Schorienmer. \\
\hline 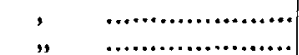 & $\mathrm{CHMe}_{2} \cdot\left(\mathrm{CH}_{2}\right)_{2} \cdot \mathrm{Me}$ & b.p. 335 & Wislicenng \\
\hline 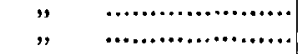 & $\begin{array}{l}\mathrm{CHMeEt} \\
\mathrm{CHMe}_{2} . \mathrm{CHMe}_{2} . \ldots\end{array}$ & $\begin{array}{l}\text { b.p. } 337 \\
\text { b.p. } 331\end{array}$ & $\begin{array}{l}\text { Wislicenus. } \\
\text { Schorlemmer. }\end{array}$ \\
\hline , $\quad$.................. & $\mathrm{OMe}_{3} \mathrm{Et} \ldots \ldots \ldots \ldots$ & b.p. 318 & Butlerow. \\
\hline Heptane.................. & Normal ..... & b.p. 371 & Thorpe. \\
\hline 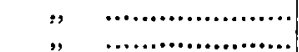 & $\mathrm{OHEt}_{3} \ldots$ & b.p. 370 & Ladenburg. \\
\hline 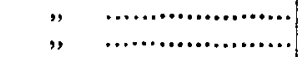 & CIIMeEtPr ${ }^{\alpha} \ldots$ & b.p. 364 & Just. \\
\hline 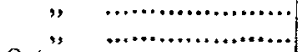 & $\begin{array}{l}\mathrm{CHMe}_{2} \cdot\left(\mathrm{CH}_{2}\right)_{2} \cdot \text { Et.. } \\
\mathrm{CMe}_{2} \mathrm{Et}, \ldots \ldots \ldots \ldots . .\end{array}$ & $\begin{array}{l}\text { b.p. } 363 \\
\text { b.p. } 360\end{array}$ & $\begin{array}{l}\text { Thorpe. } \\
\text { Friedel. }\end{array}$ \\
\hline Oetane & Normal .............. & b.p. 398 & Thorpe. \\
\hline 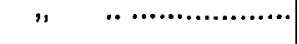 & $\mathrm{CHMe}_{2} \cdot\left(\mathrm{CH}_{2}\right)_{2} \cdot \mathrm{CHMe} \mathrm{Me}_{2}$ & b.p. 381 & Williams. \\
\hline 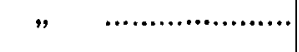 & $\mathrm{CMe}_{3}, \mathrm{CMe}_{3}$ & $\begin{array}{l}\text { b.p. } 379 \\
\text { m.p. } 369\end{array}$ & Lwow. \\
\hline Nonane .............. & Normal & $\begin{array}{r}\text { b.p. } 422 \\
\text { m.p. } 222\end{array}$ & Krafft. \\
\hline ….................. & $\mathrm{CHMe}_{2} \cdot\left(\mathrm{CH}_{2}\right)_{3} \cdot \mathrm{CHMO}_{2}$ & b.p. 405 & Wurtz. \\
\hline $\begin{array}{c}" \\
\text { Decane }\end{array}$ & $\mathrm{PrB} \cdot \mathrm{OH}_{2} \cdot \mathrm{CHM}$ & $\begin{array}{l}\text { b.p. } 403 \\
\text { b.p. } 446\end{array}$ & Silra. \\
\hline Decane & Normal ..... & m.p. 241 & Krafft. \\
\hline Undecane ...... & $"$ & $\begin{array}{l}\text { b.p. } 468 \\
\text { m.p. } 247\end{array}$ & $"$ \\
\hline Amylene & $\mathrm{CMe}_{2}$ : CHMe .. & b.p. 309 & Flawitzky. \\
\hline Hexylene & $\mathrm{CMe}_{2}: \mathrm{CMe}_{2} \ldots \ldots$ & b.p. 346 & Rizza. \\
\hline 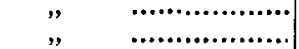 & $\mathrm{CMe}_{2}$ : CHEt....... & $\begin{array}{l}\text { b.p. } 343 \\
\text { b.p. } 339\end{array}$ & Jawein. \\
\hline Heptylene .................... & $\mathrm{CM}_{2}: \mathrm{CMeEt}$ & b.p. 366 & Kaschirsky. \\
\hline …............... & $\mathrm{CHMe}_{2}, \mathrm{CH}: \mathrm{CMe}$ & b.p. 355 & Markownikow. \\
\hline 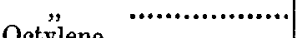 & $\mathrm{CMe}_{3}, \mathrm{CMe}: \mathrm{CH}_{2}$ & b.p. 352 & Eltekoff. \\
\hline Octylene $\quad . . . . . . . . . . . . .$. & $\mathrm{C}_{5} \mathrm{H}_{7} \mathrm{Me}_{3} \ldots \ldots \ldots$ & b.p. 395 & Williams. \\
\hline (................. & CHPr $\beta$ : & b.p. 391 & Fossek. \\
\hline Methylethyl-acetylene ... & $\begin{array}{l}\mathrm{OMe}_{2}: \mathrm{OH} . \mathrm{CMe}_{3} \ldots \ldots \ldots \\
\mathrm{OMe}: \mathrm{OEt} \ldots \ldots \ldots \ldots \ldots \ldots\end{array}$ & $\begin{array}{l}\text { b.p. } 376 \\
\text { b.p. } 324\end{array}$ & $\begin{array}{l}\text { Butlerow. } \\
\text { Eltekoff. }\end{array}$ \\
\hline Valerylene ...................... & $\mathrm{CMe}_{2}: \mathrm{O}: \mathrm{CH}_{2}$. & b.p. 318 & Reboul. \\
\hline Isopropyl-acetylene & $\mathrm{CH}$ & b.p. 301 & Flawitzky. \\
\hline 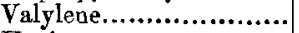 & $\mathrm{CH}_{2}: \mathrm{CMe} . \mathrm{O}$ : $\mathrm{OH}$. & b.p. 323 & Reboul. \\
\hline Hexine .... & $\mathrm{CMe} \vdots \mathrm{CPr}^{\alpha} \ldots \ldots \ldots$ & b.p. 355 & Hecht. \\
\hline Heptine ......... & CHMe : C: CHPr... & b.p. 376 & Tilden. \\
\hline ….................... & $\mathrm{CMe}_{2}: \mathrm{C}: \mathrm{CMe}_{2}$ & b.p. 343 & Henry. \\
\hline
\end{tabular}

By the normal halogen or alkyl compounds of the hydrocarbon radicals we mean the compounds formed by the following radicals with the halogens chlorine, bromine, and iodine, or with methyl, ethyl, propyl, \&c. ; viz. :- 


\begin{tabular}{|c|c|c|c|c|c|c|}
\hline & Series 1. & Series 2. & Series 3. & Series 4. & Series 5. & \\
\hline Group I .... & ....... & ....... & $\left(\mathrm{C}_{3} \mathrm{H}\right) \mathrm{X}_{1}$ & $\left(\mathrm{C}_{4} \mathrm{H}_{3}\right) \mathrm{X}_{1}$ & $\left(\mathrm{C}_{5} \mathrm{H}_{5}\right) \mathrm{X}_{1}$ & \\
\hline " II. & $\ldots$ & $\left(C_{2}\right) X_{2}$ & $\left(\mathrm{O}_{3} \mathrm{H}_{2}\right) \mathrm{X}_{2}$ & $\left(\mathrm{O}_{4} \mathrm{H}_{1}\right) \mathrm{X}_{2}$ & $\left(\mathbf{O}_{5} \mathbf{H}_{3}\right) \mathbf{X}_{2}$ & 0 \\
\hline III. & ... & $\left(\mathrm{C}_{2} \mathrm{H}\right) \mathrm{X}_{3}$ & $\left(\mathrm{C}_{3} \mathrm{H}_{3}\right) \mathrm{X}_{3}$ & $\left(\mathrm{C}_{4} \mathrm{H}_{5}\right) \mathrm{X}_{3}$ & $\left(\mathrm{C}_{\bar{a}} \mathbf{H}_{7}\right) \mathbf{X}_{3}$ & \\
\hline IV. & $\mathrm{CX}_{4}$ & $\left(\mathrm{C}_{2} \mathrm{H}_{2}\right) \mathrm{X}_{1}$ & $\left(\mathrm{C}_{3} \mathrm{H}_{4}\right) \mathrm{X}_{4}$ & $\left(\mathrm{C}_{4} \mathrm{H}_{6}\right) \mathrm{X}_{4}$ & $\left(\mathrm{C}_{5} \mathrm{H}_{8}\right) \mathrm{X}_{4}$ & $\infty$ \\
\hline V. & $(\mathrm{CH}) \mathrm{X}_{3}$ & $\left(\mathrm{O}_{2} \mathrm{H}_{3}\right) \mathbf{X}_{3}$ & $\left(\mathrm{C}_{3} \mathrm{H}_{5}\right) \mathrm{X}_{3}$ & $\left(\mathrm{C}_{4} \mathrm{H}_{7}\right) \mathrm{X}_{3}$ & $\left(\mathrm{C}_{\overrightarrow{0}} \mathrm{H}_{9}\right) \mathrm{X}_{3}$ & 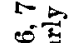 \\
\hline "VI. & $\left(\mathrm{CH}_{2}\right) \mathrm{X}_{2}$ & $\left(\mathrm{C}_{2} \mathrm{H}_{4}\right) \mathrm{X}_{2}$ & $\left(\mathrm{C}_{3} \mathrm{H}_{6}\right) \mathrm{X}_{2}$ & $\left(\mathrm{C}_{4} \mathrm{H}_{8}\right) \mathrm{X}_{2}$ & $\left(\mathrm{C}_{5} \mathrm{H}_{10}\right) \mathrm{X}_{2}$ & 总: \\
\hline " VIl... & $\left(\mathrm{CH}_{3}\right) \mathrm{X}_{1}$ & $\left(\mathrm{O}_{2} \mathrm{H}_{5}\right) \mathrm{X}_{1}$ & $\left(\mathrm{C}_{3} \mathrm{H}_{7}\right) \mathrm{X}_{1}$ & $\left(\mathrm{C}_{4} \mathrm{H}_{9}\right) \mathbf{X}$ & $\left(\mathrm{O}_{5} \mathrm{H}_{31}\right) \mathrm{X}_{1}$ & 总 \\
\hline
\end{tabular}

In the above, $\mathrm{X}=\mathrm{Cl}, \mathrm{Br}, \mathrm{I}, \mathrm{CH}_{3}, \mathrm{C}_{2} \mathrm{H}_{5}$, or $\mathrm{C}_{3} \mathrm{H}_{7}$, \&c.

The above series, of course, might be considerably extended ; but I have not thought it necessary to carry my investigations beyond the 8th series for the halogen compounds, nor beyond the 5 th series for the alkyl compounds*.

When there are several isomeric modifications of the same empirical formula, only those which are strictly analogous should be compared; thus, normal compounds should be compared with normal, and iso-compounds with iso-, \&c. In some instances, however, this is not possible, owing to the data being incomplete, or to the constitution being unknown, or when the compounds belonging to different groups of the same series are to be compared; in this case the mean of the melting- or boiling-points of the several isomeric compounds is employed. As a general rule, however, the same results are obtained, no matter whether we use the mean melting- or boiling-point for all the isomeric compounds, or whether wo compare only those compounds which are strictly analogous.

Now the same general rule that we have already shown (ibid. xviii. p. 2) to hold good as regards the corresponding compounds of the elements also holds in the case of the above hydrocarbon radicals; for in whatever way we may arrange the melting- or boiling-points of their normal halogen or alkyl compounds, provided only that we arrange them systematically, we always find that certain definite and regular relations may be traced between them. The following are examples of such relations :-

RELATION 1. If the above hydrocarbon radicals be arranged in the order of their atomic weights, then the melting-points and boiling-points of both their halogen and alkyl compounds rise and fall periodically.

This periodicity is such that the values increase from the

- The methide curve in the diagram which accompanies the paper has, however, been completed to the end of the eighth series. 
first (monad) up to the fourth or middle (tetrad) member of each series, and then diminish to the seventh (monad) or last member (see Table II.*).

This periodicity is not exactly the same as that in the case of the corresponding compounds of the elements ; I shall not, however, discuss this point at present, but defer it to my next communication.

TABLE II.-Illustrating Relations 1 and 2.

\begin{tabular}{|c|c|c|c|c|c|c|c|}
\hline & & \multicolumn{2}{|c|}{ OHLORIDES. } & \multicolumn{2}{|c|}{ Bromines. } & \multicolumn{2}{|c|}{ IoDIDES. } \\
\hline & & M.P. & B.P. & M.P. & B.P. & M.P. & B.P. \\
\hline \multirow{5}{*}{ 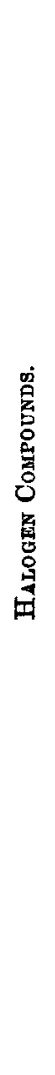 } & $\begin{array}{c}\ldots \ldots . . \\
\ldots \ldots . . \\
\ldots \ldots . \\
\ddot{\mathrm{C}}^{\mathrm{iq}} \ldots . . \\
(\mathrm{CH})^{\mathrm{iii}} \\
\left(\mathrm{CH}_{2}\right)^{\mathrm{ii}} \\
\left(\mathrm{CH}_{3}\right)^{\mathrm{i}}\end{array}$ & $\begin{array}{c}\ldots \ldots . . \\
\ldots \ldots . . \\
\ldots \ldots . \\
248 \\
203 \\
\text { Liquid. } \\
\text { Liquid. }\end{array}$ & $\begin{array}{l}\ldots \ldots . \\
\ldots \ldots . \\
\ldots \ldots . \\
350 \\
334 \\
315 \\
249\end{array}$ & $\begin{array}{l}\ldots \ldots . . \\
\ldots \ldots . \\
\ldots \ldots . \\
364 \\
281 \\
\text { b. } 261 \\
\text { Liquid. }\end{array}$ & $\begin{array}{l}\ldots \ldots . . \\
\ldots \ldots . . \\
\ldots 62 \\
424 \\
370 \\
278\end{array}$ & $\begin{array}{c}\ldots \ldots . . \\
\ldots \ldots . . \\
\ldots \ldots . \\
420 \\
392 \\
277 \\
\text { Liquid. }\end{array}$ & $\begin{array}{l}\ldots \ldots . . \\
\ldots \ldots . \\
\ldots 20 \\
\ldots \ldots . \\
453 \\
315\end{array}$ \\
\hline & $\begin{array}{c}\dddot{\mathrm{C}}_{2} \mathrm{ii} \\
\left(\mathrm{C}_{2} \mathrm{H}\right)^{\mathrm{iii}} \\
\left(\mathrm{C}_{2} \mathrm{H}_{2}\right)^{i \mathrm{iv}} \\
\left(\mathrm{C}_{2} \mathrm{H}_{3}\right)^{\mathrm{ii}} \\
\left(\mathrm{O}_{2} \mathrm{H}_{4}\right){ }^{\mathrm{ii}} \\
\left(\mathrm{C}_{2} \mathrm{H}_{5}\right)^{\mathrm{I}}\end{array}$ & 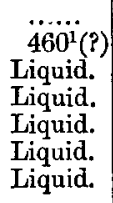 & $\begin{array}{l}460^{1}(?) \\
360 \\
411 \\
367 \\
345 \\
285\end{array}$ & $\begin{array}{l}\ldots \ldots . . \\
\ldots \ldots . . \\
\text { Liquid. } \\
327 \\
\text { Liquid. } \\
\ldots \ldots . . \\
\text { Liquid. }\end{array}$ & $\begin{array}{l}\ldots . . . \\
\ldots 35 \\
488 \\
462 \\
393 \\
312\end{array}$ & $\begin{array}{c}\ldots \ldots . . \\
\ldots \ldots . . \\
\ldots \ldots . . \\
\ldots . . . \\
\ldots \ldots . \\
355 \\
\text { Liquid. }\end{array}$ & $\begin{array}{l}\cdots \ldots . . \\
\ldots \ldots . . \\
\ldots \ldots . . \\
\cdots \ldots . . \\
\dddot{453} d . \\
\mathbf{3 4 5}\end{array}$ \\
\hline & $\begin{array}{l}\left(\mathrm{C}_{3} \mathrm{H}\right)^{\mathrm{i}} \\
\left(\mathrm{O}_{3} \mathrm{H}_{2}\right)^{\mathrm{ii}} \\
\left(\mathrm{O}_{3} \mathrm{H}_{3}\right)^{\mathrm{iii}} \\
\left(\mathrm{C}_{3} \mathrm{H}_{4}\right)^{\mathrm{iv}} \\
\left(\mathrm{O}_{3} \mathbf{H}_{3}\right)^{i \mathrm{ii}} \\
\left(\mathrm{C}_{3} \mathrm{H}_{6}\right)^{\mathrm{ij}} \\
\left(\mathrm{C}_{3} \mathrm{H}_{7}\right)^{\mathrm{i}}\end{array}$ & $\begin{array}{l}\text { Liquid. } \\
\text { Liquid. } \\
434 \\
\text { b. } 263 \\
\text { Liquid. } \\
\text { Liquid. }\end{array}$ & $\begin{array}{l}351 \\
402 \\
447 \\
415 \\
366 \\
315\end{array}$ & $\begin{array}{c}\ldots . . . \\
\ldots \ldots . . \\
\text { Liquid. } \\
405 \\
289 \\
\text { Liquid. } \\
\text { Liquid. }\end{array}$ & $\begin{array}{l}\cdots \ldots . . \\
\ldots 57 \\
513 \\
476 \\
411 \\
339\end{array}$ & $\begin{array}{c}\ldots \ldots . . \\
\dddot{326} \\
\ldots \ldots . . \\
\ldots \ldots . . . \\
\text { Liquid. } \\
\text { Liquid. }\end{array}$ & $\begin{array}{l}\ldots \ldots . . \\
\ldots \ldots . . \\
\ldots \ldots . . \\
\ldots \ldots . \\
460 \\
368\end{array}$ \\
\hline & 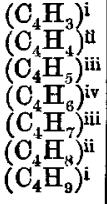 & $\begin{array}{c}\ldots \ldots . \\
\ldots \ldots . \\
\ldots \ldots . \\
346 \\
\ldots \ldots . . \\
\text { Liquid. } \\
\text { Liquid. }\end{array}$ & $\begin{array}{l}\ldots \ldots . . \\
\ldots \ldots . \\
\ldots \ldots . . \\
\ldots \ldots . \\
\ldots \ldots . \\
386 \\
339\end{array}$ & $\begin{array}{c}\ldots . . . \\
\ldots . . . \\
\ldots \ldots . \\
383 \\
\ldots \ldots . . \\
\text { Liquid. } \\
\text { Liquid. }\end{array}$ & $\begin{array}{l}\ldots . . . \\
\ldots \ldots . . \\
\ldots \ldots . . \\
\ldots \ldots . \\
487 \\
431 \\
361\end{array}$ & $\begin{array}{c}\ldots \ldots . \\
\ldots \ldots . \\
\ldots \ldots . \\
\ldots \ldots . \\
\ldots \ldots . \\
\ldots . . . \\
\text { Liquid. }\end{array}$ & $\begin{array}{l}\ldots \ldots . . \\
\ldots \ldots . . \\
\ldots \ldots . \\
\ldots \ldots . \\
\ldots \ldots . . \\
\ldots \ldots . \\
390\end{array}$ \\
\hline & 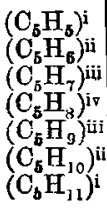 & $\begin{array}{l}\text { Liquid. } \\
\text { Liquid. } \\
\text { Liquid. } \\
\text { Liquid. } \\
\text { Liquid. } \\
\text { Liquid. } \\
\text { Liqnid. }\end{array}$ & $\begin{array}{l}566(?)^{2} \\
419 \\
\mathbf{4 7 3} \\
508 \\
454 \\
417 \\
371\end{array}$ & $\begin{array}{c}\ldots . . . \\
\ldots \ldots . . \\
\ldots \ldots . . \\
388 \\
\text { Solid. } \\
\text { Liquid. } \\
\text { Liquid. }\end{array}$ & $\begin{array}{l}\ldots \ldots . . \\
\ldots . . . \\
\ldots \ldots . \\
548 \\
\ldots \ldots . \\
453 \\
391\end{array}$ & $\begin{array}{c}\ldots . . . \\
\ldots . . . \\
\ldots . . . \\
\ldots \ldots . \\
\ldots . . . \\
\text { Liq.... } \\
\text { Liquid. }\end{array}$ & $\begin{array}{l}\ldots \ldots . \\
\ldots \ldots . \\
\ldots \ldots . \\
\ldots \ldots . \\
\ldots \ldots . \\
\ldots \ldots . \\
416\end{array}$ \\
\hline
\end{tabular}

* The periodicity is rendered still more evident by the curves for the methides, chlorides, and bromides shown in the diagram (p. 514). 
TABLe II. (continued).-Illustrating Relations 1 and 2.

\begin{tabular}{|c|c|c|c|c|c|c|c|}
\hline & & \multicolumn{2}{|c|}{ Methides. } & \multicolumn{2}{|c|}{ Etrides. } & \multicolumn{2}{|c|}{ Normal Propides. } \\
\hline & & M.P. & B.P. & M.P. & B.P. & M.P. & B.P. \\
\hline \multirow{5}{*}{ 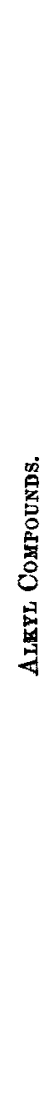 } & $\begin{array}{c}\ldots \ldots . \\
\cdots \ldots . . \\
\cdots \cdots . . \\
\mathrm{C}^{\mathrm{iv}} \ldots \\
(\mathrm{CH})^{\mathrm{iij}} \\
\left(\mathrm{CH}_{2}\right)^{\mathbf{i i}} \\
\left(\mathrm{CH}_{3}\right)^{\mathrm{i}}\end{array}$ & $\begin{array}{c}\ldots \ldots . . \\
\ldots \ldots . \\
\ldots \ldots . . \\
\text { Solidifies } \\
253 \\
\text { Liquid. } \\
\text { Liquid. } \\
\text {...... }\end{array}$ & $\begin{array}{l}\ldots \ldots . . \\
\ldots \ldots . \\
\cdots \cdots . . \\
282 \\
256 \\
245 \\
\text { Gas. }\end{array}$ & $\begin{array}{c}\ldots . . . \\
\ldots . . . \\
\ldots . . . \\
\ldots . . . \\
\text { Liquid. } \\
\text { Liquid. } \\
\text { Liquid. }\end{array}$ & $\begin{array}{l}\ldots \ldots . \\
\ldots \ldots . \\
\ldots \ldots . \\
\ldots \ldots . \\
370 \\
311 \\
245\end{array}$ & $\begin{array}{c}\ldots \ldots . \\
\ldots \ldots . . \\
\ldots \ldots . \\
\ldots . . . \\
\ldots \ldots . . \\
\text { Liquid. } \\
\text { Liquid. }\end{array}$ & $\begin{array}{l}\ldots \ldots . . \\
\ldots \ldots . \\
\cdots \ldots . . \\
\ldots \ldots . . \\
\ldots \ldots . \\
371 \\
274\end{array}$ \\
\hline & 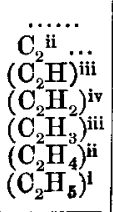 & $\begin{array}{l}\text {....... } \\
\text { Li..... } \\
\text { Liquid. } \\
\text { Liquid. } \\
\text { Liquid. } \\
\text { Liquid. } \\
\text { Liquid. }\end{array}$ & $\begin{array}{l}\ldots \ldots . \\
\ldots 309 \\
325 \\
293 \\
265 \\
245\end{array}$ & $\begin{array}{l}\ldots . . . \\
\ldots \ldots . . \\
\ldots \ldots . . \\
\ldots \ldots . . \\
\ldots \ldots . . \\
\text { Iiquid. } \\
\text { Liquid. }\end{array}$ & $\begin{array}{l}\ldots \ldots . . \\
\ldots \ldots . \\
\ldots \ldots . \\
\ldots \ldots . \\
\ldots \ldots . \\
341 \\
\mathbf{2 7 4}\end{array}$ & $\begin{array}{c}\ldots . . . \\
\ldots . . . \\
\ldots . . . \\
\cdots \cdots . . \\
\ldots \ldots . . \\
\text { Liquid. } \\
\text { Liquid. }\end{array}$ & $\begin{array}{l}\ldots \ldots . . \\
\ldots \ldots . \\
\ldots \ldots . . \\
\ldots \ldots . \\
\ldots \ldots . \\
398 \\
311\end{array}$ \\
\hline & 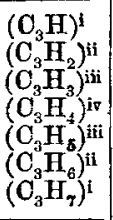 & $\begin{array}{l}\text { Liquid. } \\
\text { Liquid. } \\
\text { Liquid. } \\
\text { Liquid. } \\
\text { Liquid. } \\
\text { Liquid. }\end{array}$ & $\begin{array}{l}\mathbf{3 1 4} \\
343 \\
360 \\
330 \\
\mathbf{2 9 9} \\
\mathbf{2 6 5}\end{array}$ & $\begin{array}{l}\ldots . . . \\
\ldots \ldots . . \\
\ldots \ldots . . . \\
\ldots \ldots . . \\
\text { Liquid. } \\
\text { Liquid. } \\
\end{array}$ & $\begin{array}{l}\ldots \ldots . \\
\ldots \ldots . \\
\ldots \ldots . \\
\ldots \ldots . \\
\ldots \ldots . \\
366 \\
307\end{array}$ & $\begin{array}{c}\ldots \ldots . . \\
\ldots \ldots . . \\
\ldots \ldots . \\
\ldots \ldots . . \\
\ldots \ldots . \\
222 \\
\text { Liquid. }\end{array}$ & $\begin{array}{l}\ldots \ldots . \\
\ldots \ldots . \\
\ldots \ldots . \\
\ldots \ldots . \\
\ldots \ldots . \\
422 \\
340\end{array}$ \\
\hline & $\begin{array}{l}\left(\mathrm{C}_{4} \mathrm{H}_{3}\right)^{\mathrm{i}} \\
\left(\mathrm{C}_{4} \mathrm{H}_{4}\right)^{\mathrm{ii}} \\
\left(\mathrm{C}_{4} \mathrm{H}_{5}\right)^{\mathrm{iii}} \\
\left(\mathrm{O}_{4} \mathrm{H}_{6}\right)^{\mathrm{iv}} \\
\left(\mathrm{C}_{4} \mathrm{H}_{7}{ }^{\mathrm{iii}}\right. \\
\left(\mathrm{C}_{4} \mathrm{H}_{8}\right)^{\mathrm{ii}} \\
\left(\mathrm{C}_{4} \mathrm{H}_{9}\right)^{\mathrm{i}}\end{array}$ & $\begin{array}{l}\text { Liquid. } \\
\text { Liquid. } \\
\text { Liquid. } \\
\text { 369 } \\
\text { Liquid. } \\
\text { Liquid. } \\
\text { Liquid. }\end{array}$ & $\begin{array}{l}323 \\
355 \\
358 \\
380 \\
364 \\
333 \\
299\end{array}$ & $\begin{array}{l}\ldots . . . \\
\ldots . . . \\
\ldots . . . \\
\text {........ } \\
\text { Liquid. } \\
\text { Liquid. }\end{array}$ & $\begin{array}{l}\ldots \ldots . . \\
\ldots \ldots . . \\
\ldots \ldots . . \\
\ldots \ldots . . \\
\ddot{3} . . . \\
338\end{array}$ & $\begin{array}{c}\ldots . . . \\
\ldots . . . \\
\ldots . . . \\
\ldots \ldots . . \\
\ldots \ldots . . \\
241 \\
\text { Liquid. }\end{array}$ & $\begin{array}{l}\ldots \ldots . . \\
\ldots \ldots . . \\
\ldots \ldots . . \\
\ldots \ldots . . \\
\ldots \ldots . \\
446 \\
366\end{array}$ \\
\hline & $\begin{array}{l}\left(\mathrm{C}_{5} \mathrm{H}_{6}\right)^{\mathrm{i}} \\
\left(\mathrm{C}_{5} \mathrm{H}_{6}\right)^{\mathrm{ii}} \\
\left(\mathrm{C}_{5} \mathrm{H}_{5}\right)^{\mathrm{iii}} \\
\left(\mathrm{C}_{5} \mathrm{H}_{8}\right)^{\mathrm{iv}} \\
\left(\mathrm{C}_{6} \mathrm{H}_{9}\right)^{\mathrm{iij}} \\
\left(\mathrm{C}_{6} \mathrm{H}_{10}\right)^{-i \mathrm{i}} \\
\left(\mathrm{C}_{6} \mathrm{H}_{11}\right)^{\mathrm{i}}\end{array}$ & $\begin{array}{l}\text { Liquid. } \\
\text { Liquid. } \\
\text { Liquid. } \\
\text { Liquid. } \\
\text { Liquid. } \\
\text { Liquid. }\end{array}$ & $\begin{array}{l}360 \\
387 \\
404 \\
381 \\
365 \\
333\end{array}$ & $\begin{array}{c}\ldots . . . \\
\ldots \ldots . . \\
\ldots \ldots . . \\
\ldots \ldots . \\
\dddot{20 .} \\
\text { Liquid. }\end{array}$ & $\begin{array}{l}\ldots \ldots . . \\
\ldots \ldots . . \\
\ldots \ldots . . \\
\ldots \ldots . . \\
\dddot{422} \\
365\end{array}$ & 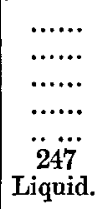 & $\begin{array}{l}\ldots \ldots . . \\
\ldots \ldots . . \\
\ldots \ldots . . \\
\ldots \ldots . \\
\ldots \ldots . \\
468 \\
398\end{array}$ \\
\hline
\end{tabular}

N.B.-In the above table the mean value is given when there are several isomers having the same empirical composition. Sometimes, however, in the case of the melting-points, there are several pairs or triplets of isomerie substances of which the melting-point of one only is known, the others being liquid; a mean number is therefore impossible; in this case the known melting-point is given.

1 This is the mean of $448^{\circ}-473^{\circ}$, which are the temperatures given in Watts's Dictionary (i. p. 768) as those between which protochloride of carbon melts and boils. Chlorine does not act upon this compound even in sunshine. It is most probably a polymer $\left(\mathrm{C}_{2} \mathrm{Cl}_{2}\right)_{n}$. Berthelot in fact regards it as $\mathrm{C}_{10} \mathrm{Cl}_{10}$. Its vapour-density, however, does not appear to have been determined.

2 'This is the boiling-point of chlornicene, as given by St. Evre (Jahresbericht, i. p. 530). This substance, if a definite compound, is probably a polymer $\left(\mathrm{C}_{5} \mathrm{H}_{5} \mathrm{Cl}\right)_{n}$. 
ReLation 2. The melting-points and boiling-points of either the halogen or alkyl compounds of any hydrocarbon radical increase as we pass from the chloride to the bromide and thence to the iodide; or from the methide to the ethide, and thence to the propide, \&c. (See Tables II. and III.)

TABLE III.-Illustrating Relations 2 and 3.

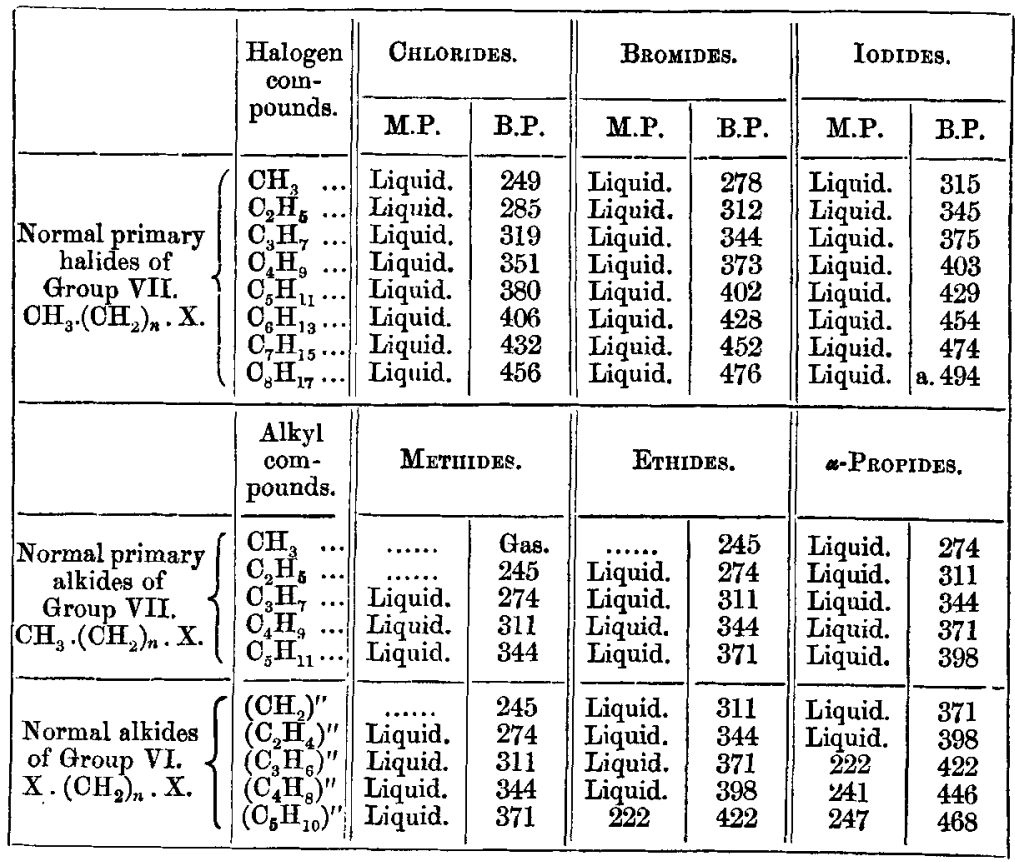

RELATION 3. The melting-and boiling-points of either the halogen or alkyl compounds of the hydrocarbon radicals of any one group increase as the atomic weight of the hydrocarbon radical increases. (See Table III.)

ReLation 4. The differences between the boiling-points (and also between the melting-points) of the chlorides and bromides, bromides and iodides, chlorides and iodides (or between those of the methides and ethides, ethides and propides, propides and butides, \&c.), increase algebraically from the first member of each series of hydrocarbon radicals up to the fourth or middle (tetrad) 
member and then diminish to the seventh or last member. (Seo Table IV.)

TABLE IV.-Illustrating Relations 4 and 5.

\begin{tabular}{|c|c|c|c|c|c|c|}
\hline & $\mathrm{Br}-\mathrm{Cl}$ & $\mathbf{I}-\mathbf{B r}$. & $\mathrm{I}-\mathrm{Cl}$. & $\mathrm{Et}-\mathrm{Me}$ & Pr-Et. & $\mathrm{Pr}-\mathrm{Me}$ \\
\hline \multirow[t]{2}{*}{$\overline{\text { Diff. in at. wts. }}$} & $44 \cdot 5$ & 47 & $91 \cdot 5$ & 14 & 14 & 28 \\
\hline & B.P. & B.P. & B.P. & B.P. & B.P. & B.P. \\
\hline 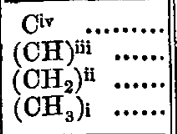 & $\begin{array}{r}112 \\
90 \\
55 \\
28\end{array}$ & $\begin{array}{l}158 \\
\ldots \ldots \\
83 \\
39\end{array}$ & $\begin{array}{r}270 \\
13 \% \\
66\end{array}$ & $\begin{array}{c}1114 \\
66 \\
\cdots \cdots\end{array}$ & $\begin{array}{l}\cdots \ldots . \\
\ldots \ldots \\
29\end{array}$ & $\begin{array}{l}\ldots \ldots . \\
126 \\
\ldots \ldots .\end{array}$ \\
\hline 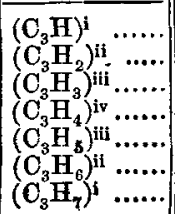 & $\begin{array}{c}\ldots \ldots . \\
\ldots \ldots . \\
55 \\
66 \\
61 \\
45 \\
24\end{array}$ & $\begin{array}{c}\ldots \ldots . \\
\ldots \ldots . \\
\ldots \ldots . \\
\ldots \ldots . \\
\ldots \ldots . \\
42 \\
29\end{array}$ & $\begin{array}{c}\ldots \ldots . . \\
\ldots \ldots . . \\
\ldots \ldots . . \\
\ldots \ldots . . \\
\ldots \ldots . . \\
94 \\
53\end{array}$ & $\begin{array}{c}\ldots \ldots . . \\
\ldots \ldots . \\
\ldots \ldots . . \\
\ldots \ldots . \\
\ldots \ldots \\
37\end{array}$ & $\begin{array}{c}\ldots . . . \\
\ldots \ldots . \\
\ldots \ldots . . \\
\ldots \ldots . . \\
\ldots \ldots . \\
51 \\
33\end{array}$ & $\begin{array}{c}\ldots . . . \\
\ldots \ldots . \\
\ldots \ldots . . \\
\ldots \ldots . \\
\ldots \ldots . . \\
111 \\
70\end{array}$ \\
\hline
\end{tabular}

The number of cases in which Relation 4 can be applied is unfortunately very limited; but, in so far as it is applicable, the data obey exactly the same rule as those of the halogen and alkyl compounds of the elements.

ReLation 5. The differences referred to in (4) increase as the difference between the atomic weights of the halogens (or of the alkyl radicals) inereases. In the case where the difference between the alkyl radicals is the same, the differences between the boiling-points (or melting-points) diminish as the sum of the atomic weights of the several pairs of alkyl radicals increases. (See Tables IV. and V.)

ReLation 6. The differences referred to in (4) diminish algebraically as the atomic weight of the hydroearbon radical increases. (See Table V.) 
TABLE V.-Illustrating Relations 5 and 6.

\begin{tabular}{|c|c|c|c|c|c|c|c|}
\hline & & $\mathrm{Br}-\mathrm{Cl}$ & $\mathrm{I}-\mathrm{Br}$. & $\mathrm{I}-\mathrm{Cl}$. & Et-Me. & $P r-E t$. & $\mathrm{Pr}-\mathrm{Me}$ \\
\hline & $\begin{array}{l}\text { Diff. in } \\
\text { at. wts. }\end{array}$ & $44 \cdot 5$ & 47 & 91.5 & 14 & 14 & 28 \\
\hline & & B.P. & B.P. & B.P. & B.P. & B.P. & B.P. \\
\hline $\begin{array}{c}\text { Normal halides } \\
\text { and alkides of } \\
\text { Group VII. } \\
\mathrm{CH}_{3} \cdot\left(\mathrm{CH}_{2}\right)_{n} \cdot \bar{X} .\end{array}$ & \begin{tabular}{ll|}
$\mathrm{OH}_{3}$ & $\ldots$ \\
$\mathrm{C}_{2} \mathrm{H}_{5}$ & $\ldots$ \\
$\mathrm{O}_{3} \mathrm{H}_{7}$ & $\ldots$ \\
$\mathrm{C}_{4} \mathrm{H}_{9}$ & $\ldots$ \\
$\mathrm{O}_{5} \mathrm{H}_{11}$ & $\ldots$ \\
$\mathrm{C}_{6} \mathrm{H}_{13}$ & $\ldots$ \\
$\mathrm{C}_{7} \mathrm{H}_{15}$ & $\ldots$ \\
$\mathrm{C}_{8} \mathrm{H}_{17}$ & $\ldots$ \\
\end{tabular} & $\begin{array}{l}29 \\
27 \\
25 \\
22 \\
22 \\
22 \\
20 \\
20\end{array}$ & $\begin{array}{l}37 \\
33 \\
31 \\
30 \\
27 \\
26 \\
22 \\
\ldots \ldots \\
\end{array}$ & $\begin{array}{c}66 \\
60 \\
56 \\
52 \\
49 \\
48 \\
42 \\
\ldots \ldots .\end{array}$ & $\begin{array}{l}29^{*} \\
37 \\
33 \\
27 \\
27 \\
24 \\
24\end{array}$ & $\begin{array}{l}29 * \\
37 \\
33 \\
27 \\
27 \\
24 \\
24 \\
22\end{array}$ & $\begin{array}{l}66 * \\
70 \\
60 \\
54 \\
51 \\
48 \\
46\end{array}$ \\
\hline $\begin{array}{c}\text { Normal halides } \\
\text { and alkides of } \\
\text { Group VI. } \\
\mathrm{X} .\left(\mathrm{OH}_{2}\right)_{n} \cdot \mathrm{X} .\end{array}$ & 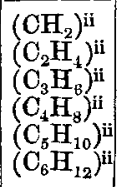 & $\begin{array}{l}55 \\
48 \\
45 \\
45 \\
36 \\
23\end{array}$ & $\begin{array}{r}83 \\
\text { a. } 60 \\
49 \\
\ldots \ldots . \\
\ldots \ldots . \\
\ldots \ldots . \\
\ldots . .\end{array}$ & $\begin{array}{r}138 \\
\text { a. } 108 \\
94 \\
\ldots \ldots . . \\
\cdots \ldots . . \\
\ldots \ldots .\end{array}$ & $\begin{array}{l}66 * \\
70 \\
60 \\
54 \\
51 \\
48\end{array}$ & $\begin{array}{l}61 \\
54 \\
51 \\
48 \\
46 \\
41\end{array}$ & $\begin{array}{r}124 \\
124 \\
111 \\
102 \\
97 \\
89\end{array}$ \\
\hline
\end{tabular}

- These four exceptions are all due to the boiling-point of propane, $\mathrm{C}_{3} \mathrm{H}_{8}$, being taken too high. The number used is based on the data given by Lefebvre, who states that propane is condensed to a liquid at $-25^{\circ}$ to $-30^{\circ} \mathrm{C}$.

ReLation 7. (a) The differences between the boiling-points (and also between the melting-points) of the halogen (or of the alkyl) compounds of the hydrocarbon radicals of each group diminish as we pass from the chlorides to the bromides, and thence to the iodides (or from the metrides to the ethides, and thence to the propides, \&c.). (See Table VI.)

(b) These differences also increase as the difference between the atomic weights of each pair of hydrocarbon radicals increases. (See Table VI.)

In those cases where there is a common difference between the atomic weights of several pairs of hydrocarbon radicals, then the differences between the boiling-points increase as the sum of the atomic weights of the several pairs of hydrocarbon radicals diminishes. (See Table VI.) 
TABLE VI.-Illustrating Relations $7 a \& b$.

\begin{tabular}{|c|c|c|c|c|c|c|c|}
\hline Group VII. & $X=$ & $\begin{array}{l}\text { Ohlo- } \\
\text { rides. }\end{array}$ & $\begin{array}{l}\text { Bro- } \\
\text { mides. }\end{array}$ & Iodides. & $\underset{\text { thides. }}{\text { Me- }}$ & Ethides. & $\begin{array}{l}\text { Pro- } \\
\text { pides. }\end{array}$ \\
\hline $\mathrm{CH}_{3} \cdot\left(\mathrm{CH}_{2}\right)_{n} \cdot \mathrm{X}$ & $\begin{array}{l}\text { Diff. in } \\
\text { at. wts. }\end{array}$ & B.P. & B.P. & B.P. & B.P. & B.P. & B.P. \\
\hline 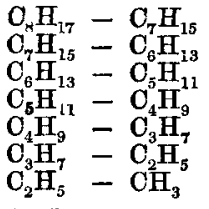 & $\left\{\begin{array}{l}\mathrm{CH}_{2} \\
=14\end{array}\right.$ & $\begin{array}{l}24 \\
26 \\
26 \\
29 \\
32 \\
34 \\
36\end{array}$ & $\begin{array}{l}24 \\
24 \\
26 \\
29 \\
29 \\
32 \\
34\end{array}$ & $\begin{array}{l}20 \\
20 \\
25 \\
26 \\
28 \\
30 \\
30\end{array}$ & $\begin{array}{l}24 \\
27 \\
27 \\
33 \\
37 \\
29^{*} \\
\cdots\end{array}$ & $\begin{array}{l}24 \\
24 \\
27 \\
27 \\
33 \\
37 \\
29^{*}\end{array}$ & $\begin{array}{l}21 \\
24 \\
\mathbf{2 4} \\
\mathbf{2 7} \\
27 \\
33 \\
37\end{array}$ \\
\hline $\begin{array}{l}\mathbf{C}_{8} \mathbf{H}_{17}-\mathbf{O}_{6} \mathbf{H}_{13} \\
\mathbf{C}_{7} \mathbf{H}_{15}-\mathrm{C}_{5} \mathbf{H}_{1 \mathrm{t}} \\
\mathbf{C}_{6} \mathbf{H}_{13}-\mathrm{C}_{4} \mathbf{H}_{9} \\
\mathbf{C}_{5} \mathbf{H}_{11}=\mathbf{C}_{3} \mathbf{H}_{7} \\
\mathbf{C}_{4} \mathbf{H}_{9}=\mathbf{C}_{2} \mathbf{H}_{5} \\
\mathbf{C}_{3} \mathbf{H}_{7}-\mathbf{C H}_{3}\end{array}$ & $\begin{array}{l}2 \mathrm{CH}_{2} \\
=28\end{array}$ & $\begin{array}{l}50 \\
52 \\
55 \\
61 \\
66 \\
70\end{array}$ & $\begin{array}{l}48 \\
50 \\
55 \\
58 \\
61 \\
66\end{array}$ & $\begin{array}{l}40 \\
45 \\
51 \\
54 \\
58 \\
60\end{array}$ & $\begin{array}{l}51 \\
54 \\
60 \\
70 \\
66^{*} \\
\cdots\end{array}$ & $\begin{array}{l}48 \\
51 \\
54 \\
60 \\
70 \\
66^{*}\end{array}$ & $\begin{array}{l}45 \\
48 \\
51 \\
54 \\
60 \\
70\end{array}$ \\
\hline $\begin{array}{l}\mathbf{C}_{8} \mathbf{H}_{17}-\mathbf{C}_{5} \mathrm{H}_{11} \\
\mathbf{C}_{7} \mathbf{H}_{15}-\mathbf{C}_{4} \mathbf{H}_{9} \\
\mathbf{C}_{6} \mathbf{H}_{13}-\mathbf{C}_{3} \mathbf{H}_{7} \\
\mathbf{C}_{5} H_{11}=\mathbf{O}_{2} H_{5} \\
\mathbf{C}_{4} \mathbf{H}_{9}-\mathrm{CH}_{3}\end{array}$ & \} $\begin{array}{l}3 \mathrm{CH}_{2} \\
=42\end{array}$ & $\begin{array}{r}76 \\
81 \\
87 \\
95 \\
102\end{array}$ & $\begin{array}{l}74 \\
79 \\
84 \\
90 \\
95\end{array}$ & $\begin{array}{l}65 \\
71 \\
79 \\
84 \\
88\end{array}$ & $\begin{array}{l}78 \\
87 \\
97 \\
99\end{array}$ & $\begin{array}{l}75 \\
78 \\
87 \\
97 \\
99\end{array}$ & $\begin{array}{l}70 \\
75 \\
78 \\
87 \\
97\end{array}$ \\
\hline $\begin{array}{l}\mathrm{C}_{8} \mathrm{H}_{17}-\mathrm{C}_{4} \mathrm{H}_{9} \\
\mathrm{C}_{7} \mathrm{H}_{15}=\mathrm{C}_{8} \mathbf{H}_{7} \\
\mathrm{C}_{6} \mathrm{H}_{13}=\mathrm{O}_{2} \mathbf{H}_{5} \\
\mathrm{C}_{5} \mathrm{H}_{11}-\mathrm{CH}_{3}\end{array}$ & $\begin{array}{l}4 \mathrm{CH}_{2} \\
=56\end{array}$ & $\begin{array}{l}105 \\
113 \\
121 \\
131\end{array}$ & $\begin{array}{l}103 \\
108 \\
116 \\
124\end{array}$ & $\begin{array}{r}91 \\
99 \\
109 \\
114\end{array}$ & $\begin{array}{r}111 \\
124 \\
126 \\
\cdots\end{array}$ & $\begin{array}{l}102 \\
111 \\
124 \\
126\end{array}$ & $\begin{array}{r}97 \\
102 \\
111 \\
124\end{array}$ \\
\hline $\begin{array}{l}\mathrm{C}_{8} \mathbf{H}_{17}-\mathbf{C}_{3} \mathbf{H}_{7} \\
\mathrm{C}_{7} \mathbf{H}_{15}=\mathbf{C}_{2} \mathbf{H}_{5} \\
\mathrm{C}_{6} \mathbf{H}_{13}-\mathbf{C H}_{3}\end{array}$ & \}$=7 \mathrm{CH}^{2}$ & $\begin{array}{l}137 \\
147 \\
157\end{array}$ & $\begin{array}{l}132 \\
140 \\
150\end{array}$ & $\begin{array}{l}119 \\
129 \\
139\end{array}$ & $\begin{array}{r}148 \\
153 \\
\ldots\end{array}$ & $\begin{array}{l}135 \\
148 \\
153\end{array}$ & $\begin{array}{l}124 \\
135 \\
148\end{array}$ \\
\hline $\begin{array}{l}\mathrm{O}_{8} \mathrm{H}_{17}-\mathrm{C}_{2} \mathrm{H}_{5} \\
\mathrm{C}_{7} \mathrm{H}_{15}-\mathrm{CH}_{3}\end{array}$ & \}$=84^{6 \mathrm{CH}_{2}}$ & $\begin{array}{l}171 \\
183\end{array}$ & $\begin{array}{l}164 \\
174\end{array}$ & $\begin{array}{l}149 \\
159\end{array}$ & $\begin{array}{r}177 \\
\ldots\end{array}$ & $\begin{array}{l}172 \\
177\end{array}$ & $\begin{array}{l}156 \\
172\end{array}$ \\
\hline $\mathbf{C}_{8} \mathbf{H}_{17}-\mathbf{C H}_{3}$ & $\begin{array}{l}7 \mathrm{CH}_{2} \\
=98\end{array}$ & 207 & 198 & 179 & $\ldots$ & 201 & 193 \\
\hline
\end{tabular}

* These exceptions are all due to the boiling-point of propane, $\mathrm{C}_{3} \mathrm{H}_{8}$, being too high; see footnote to Table V.

RELATION 8. The differences between the boiling-points $\uparrow$ of the halogen or of the alkyl compounds of the hydrocarbon radicals of the seventh group, and those of the hydrocarbon radicals of the other groups respectively, increase algebraically from the chlorides to the bromides, and thence to the iodides (or from the methides to the ethides, and thence to the propides, \& 8 .). (See Table VII.)

† Data are wanting as regards melling-points. 
Table VII.-Illustrating Relations 8 and 9.

\begin{tabular}{|c|c|c|c|c|c|c|c|c|c|}
\hline \multirow{2}{*}{ 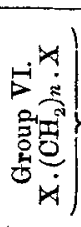 } & \multirow{2}{*}{$\operatorname{minus}$} & \multirow{2}{*}{ 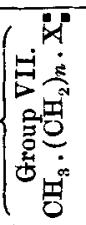 } & \multirow{2}{*}{$\begin{array}{c}X= \\
\begin{array}{c}\text { Diff. } \\
\text { in at. } \\
\text { wts. }\end{array}\end{array}$} & \multirow[t]{2}{*}{$\begin{array}{l}\text { Chlo- } \\
\text { rides. }\end{array}$} & \multirow[t]{2}{*}{$\begin{array}{l}\text { Bro- } \\
\text { mides. }\end{array}$} & \multirow[t]{2}{*}{ Iodides. } & \multirow{2}{*}{$\frac{\begin{array}{c}\text { Me- } \\
\text { thides. }\end{array}}{\text { B.P. }}$} & \multirow{2}{*}{ Ethides. } & \multirow{2}{*}{$\mid \begin{array}{c}\text { Propides } \\
\text { B.P. }\end{array}$} \\
\hline & & & & & & & & & \\
\hline $\mathrm{CH}_{2}$ & - & $\mathrm{O}_{8} \mathrm{H}_{17}$ & -99 & -141 & -106 & $6 .-41$ & -177 & -135 & -97 \\
\hline $\begin{array}{l}\mathrm{CH}_{2} \\
\mathrm{O}_{2} \mathrm{H}_{4}\end{array}$ & - & $\begin{array}{l}\mathbf{C}_{7} \mathbf{H}_{15} \\
\mathbf{C}_{8} \mathbf{H}_{17}\end{array}$ & \}-85 & $\begin{array}{l}-117 \\
-99\end{array}$ & $\begin{array}{l}-82 \\
-71\end{array}$ & $\begin{array}{l}-21 \\
\ldots \ldots .\end{array}$ & $\begin{array}{l}-153 \\
-148\end{array}$ & $\begin{array}{l}-111 \\
-102\end{array}$ & $\begin{array}{l}-75 \\
-70\end{array}$ \\
\hline $\begin{array}{l}\mathrm{OH}_{2} \\
\mathrm{CH}_{2} \mathrm{H}_{4} \\
\mathrm{O}_{3} \mathrm{H}_{6}\end{array}$ & I & $\begin{array}{l}\mathrm{C}_{6} \mathbf{H}_{13} \\
\mathrm{C}_{7} \mathbf{H}_{15} \\
\mathrm{O}_{8} \mathbf{H}_{17}\end{array}$ & -71 & $\begin{array}{l}-91 \\
-75 \\
-64\end{array}$ & $\begin{array}{l}-58 \\
=47 \\
-38\end{array}$ & $\begin{array}{c}-1 \\
\ldots \ldots \\
b .+6 d .\end{array}$ & $\begin{array}{l}-126 \\
-124 \\
-111\end{array}$ & $\begin{array}{l}-87 \\
=78 \\
-75\end{array}$ & $\begin{array}{l}=51 \\
=48 \\
-46\end{array}$ \\
\hline $\begin{array}{l}\mathrm{OH}_{2} \\
\mathrm{O}_{2} \mathrm{H}_{4} \\
\mathrm{O}_{3} \mathrm{H}_{6} \\
\mathrm{O}_{4} \mathbf{H}_{8}\end{array}$ & $\begin{array}{l}- \\
- \\
-\end{array}$ & $\begin{array}{l}\mathbf{C}_{5} \mathbf{H}_{11} \\
\mathbf{O}_{6} \mathbf{H}_{13} \\
\mathbf{C}_{7} \mathbf{H}_{15} \\
\mathbf{C}_{8} \mathbf{H}_{17}\end{array}$ & -57 & $\begin{array}{c}-65 \\
-49 \\
-\quad 39 \\
\ldots \ldots\end{array}$ & $\begin{array}{l}-32 \\
-23 \\
-14 \\
\ldots \ldots\end{array}$ & $\begin{array}{c}+24 \\
\ldots \ldots \\
+26 d \\
\ldots \ldots\end{array}$ & $\begin{array}{l}-99 \\
-97 \\
-87 \\
-78\end{array}$ & $\begin{array}{l}-60 \\
-54 \\
-51 \\
-48\end{array}$ & $\begin{array}{l}-27 \\
-24 \\
-24 \\
-22\end{array}$ \\
\hline $\begin{array}{l}\mathrm{CH}_{2} \\
\mathrm{C}_{2} \mathbf{H}_{4} \\
\mathrm{C}_{3} \mathrm{H}_{8} \\
\mathrm{C}_{4} \mathbf{H}_{8} \\
\mathrm{C}_{5} \mathrm{H}_{10}\end{array}$ & $\begin{array}{l}- \\
- \\
-\end{array}$ & $\begin{array}{l}\mathbf{C}_{4} \mathbf{H}_{9} \\
\mathbf{C}_{5} \mathbf{H}_{11} \\
\mathbf{C}_{6} \mathbf{H}_{13} \\
\mathbf{C}_{7} \mathbf{H}_{15} \\
\mathbf{C}_{8} \mathbf{H}_{17}\end{array}$ & & $\begin{array}{l}-36 \\
-23 \\
-13 \\
\ldots \ldots . \\
\ldots \ldots\end{array}$ & $\begin{array}{r}-3 \\
+\quad 3 \\
+\quad 10 \\
\cdots \cdots \\
\cdots \cdots\end{array}$ & $\begin{array}{c}+50 \\
\ldots \ldots \\
+46 d \\
\ldots \ldots \\
\ldots \ldots\end{array}$ & $\begin{array}{l}-66^{*} \\
-70 \\
=60 \\
=54 \\
-41\end{array}$ & $\begin{array}{l}-33 \\
-27 \\
-27 \\
-24 \\
-24\end{array}$ & $\begin{array}{l}0 \\
0 \\
0 \\
0 \\
0\end{array}$ \\
\hline $\begin{array}{l}\mathrm{CH}_{2} \\
\mathrm{C}_{2} \mathrm{H}_{4} \\
\mathrm{O}_{3} \mathrm{H}_{6} \\
\mathrm{C}_{4} \mathrm{H}_{8} \\
\mathrm{C}_{\tilde{n}} \mathrm{H}_{10}\end{array}$ & $\begin{array}{l}- \\
z \\
z\end{array}$ & $\begin{array}{l}\mathrm{C}_{3} \mathrm{H}_{7} \\
\mathrm{C}_{4} \mathbf{H}_{3} \\
\mathrm{C}_{5} \mathrm{H}_{11} \\
\mathrm{C}_{6} \mathrm{H}_{13} \\
\mathrm{C}_{7} \mathbf{H}_{15}\end{array}$ & -29 & $\begin{array}{r}-4 \\
+\quad 6 \\
+\quad 13 \\
\ldots \ldots \\
\ldots \ldots .\end{array}$ & $\begin{array}{l}+26 \\
+32 \\
+36 \\
\ldots \ldots \\
\cdots \cdots\end{array}$ & $\begin{array}{c}+78 \\
\ldots \ldots \\
+71 d \\
\ldots \ldots \\
\ldots \ldots\end{array}$ & $\begin{array}{l}=29^{*} \\
=37 \\
=33 \\
=27 \\
-27\end{array}$ & $\begin{array}{l}0 \\
0 \\
0 \\
0 \\
0\end{array}$ & $\begin{array}{l}+28 \\
+27 \\
+24 \\
+24 \\
+22\end{array}$ \\
\hline $\begin{array}{l}\mathrm{CH} \\
\mathrm{C}_{2} \mathrm{H}_{4} \\
\mathrm{C}_{3} \mathrm{H}_{8} \\
\mathrm{O}_{4} \mathrm{H}_{8} \\
\mathrm{C}_{5} \mathrm{H}_{10}\end{array}$ & $\begin{array}{l}- \\
\bar{z} \\
-\end{array}$ & $\begin{array}{l}\mathrm{O}_{2} \mathbf{H}_{5} \\
\mathrm{C}_{3} \mathbf{H}_{7} \\
\mathbf{C}_{4} \mathbf{H}_{9} \\
\mathrm{C}_{5} \mathbf{H}_{2]} \\
\mathbf{C}_{6} \mathbf{H}_{13}\end{array}$ & 15 & $\begin{array}{l}+30 \\
+\quad 38 \\
+\quad 42 \\
\ldots \ldots . \\
\ldots \ldots .\end{array}$ & $\begin{array}{l}+58 \\
+61 \\
+65 \\
\cdots \cdots \\
\cdots \cdots\end{array}$ & $\begin{array}{c}+108 \\
\ldots \ldots \\
+97 d \\
\ldots \ldots \\
\ldots \ldots\end{array}$ & $\begin{array}{l}0 \\
0 \\
0 \\
0 \\
0\end{array}$ & $\begin{array}{l}+37 \\
+33 \\
+27 \\
+27 \\
+24\end{array}$ & $\begin{array}{l}+61 \\
+54 \\
+51 \\
+48 \\
+46\end{array}$ \\
\hline $\begin{array}{l}\mathrm{CH}_{2} \\
\mathrm{O}_{2} \mathrm{H}_{4} \\
\mathrm{C}_{3} \mathrm{H}_{6} \\
\mathrm{O}_{4} \mathrm{H}_{8} \\
\mathrm{C}_{5} \mathrm{H}_{10}\end{array}$ & $\begin{array}{l}\overline{-} \\
\bar{z} \\
\end{array}$ & $\begin{array}{l}\mathrm{OH}_{3} \\
\mathrm{C}_{2} \mathbf{H}_{5} \\
\mathrm{C}_{3} \mathbf{H}_{7} \\
\mathrm{O}_{4} \mathbf{H}_{9} \\
\mathrm{C}_{5} \mathbf{H}_{11}\end{array}$ & 1 & $\begin{array}{c}+66 \\
+\quad 72 \\
+\quad 74 \\
\ldots \ldots . \\
\ldots \ldots .\end{array}$ & $\begin{array}{l}+92 \\
+93 \\
+94 \\
\ldots \ldots . . \\
\ldots \ldots . .\end{array}$ & $\begin{array}{c}+138 \\
\ldots \ldots \\
+125 d \\
\ldots \ldots \\
\ldots \ldots\end{array}$ & $\begin{array}{l}\dddot{+} 29 * \\
+37 \\
+33 \\
+27\end{array}$ & $\begin{array}{l}+66^{*} \\
+70 \\
+60 \\
+54 \\
+51\end{array}$ & $\begin{array}{l}+98 \\
+87 \\
+78 \\
+75 \\
+69\end{array}$ \\
\hline $\begin{array}{l}\mathrm{O}_{2} \mathrm{H}_{4} \\
\mathrm{O}_{3} \mathrm{H}_{6} \\
\mathrm{O}_{4} \mathrm{H}_{8} \\
\mathrm{O}_{5} \mathrm{H}_{10}\end{array}$ & $\begin{array}{l}\overline{-} \\
\overline{-}\end{array}$ & $\begin{array}{l}\mathrm{CH}_{3} \\
\mathrm{C}_{2} \mathbf{H}_{5} \\
\mathrm{C}_{3} \mathbf{H}_{7} \\
\mathrm{C}_{4} \mathbf{H}_{9}\end{array}$ & 13 & $\begin{array}{l}+108 \\
+108 \\
\ldots \ldots \\
\ldots \ldots\end{array}$ & $\begin{array}{l}+127 \\
+126 \\
\ldots \ldots \\
\ldots \ldots\end{array}$ & $\begin{array}{c}+155 d \\
\ldots \ldots \\
\cdots \cdots\end{array}$ & $\begin{array}{l}\dddot{1} 66^{*} \\
+70 \\
+60\end{array}$ & $\begin{array}{l}+99 \\
+97 \\
+87 \\
+78\end{array}$ & $\begin{array}{l}+124 \\
+111 \\
+102 \\
+96\end{array}$ \\
\hline $\begin{array}{l}\mathrm{C}_{3} \mathrm{H}_{6} \\
\mathrm{C}_{4} \mathrm{H}_{8} \\
\mathrm{C}_{5} \mathrm{H}_{10}\end{array}$ & $=$ & $\begin{array}{l}\mathrm{CH}_{3} \\
\mathrm{C}_{2} \mathbf{H}_{5} \\
\mathbf{C}_{3} \mathbf{H}_{7}\end{array}$ & +27 & $\begin{array}{l}+144 \\
\ldots \ldots \\
\cdots \cdots\end{array}$ & $\begin{array}{l}+160 \\
\ldots \ldots \\
\cdots \cdots \\
\end{array}$ & $\begin{array}{c}+185 d \\
\ldots \ldots \\
\ldots \ldots\end{array}$ & $\begin{array}{r}\ldots 9 \\
+99 \\
+94\end{array}$ & $\begin{array}{l}+126 \\
+124 \\
+111\end{array}$ & $\begin{array}{l}+148 \\
+135 \\
+123\end{array}$ \\
\hline $\begin{array}{l}\mathbf{C}_{4} \mathbf{H}_{8} \\
\mathbf{C}_{5} \mathbf{H}_{10}\end{array}$ & - & $\begin{array}{l}\mathrm{CH}_{3} \\
\mathrm{C}_{2} \mathrm{H}_{5}\end{array}$ & (1) & ...... & …... & …... & $\dddot{+126}$ & $\begin{array}{l}+153 \\
+148\end{array}$ & $\begin{array}{l}+172 \\
+156\end{array}$ \\
\hline $\mathrm{C}_{5} \mathbf{H}_{10}$ & - & $\mathrm{CH}_{3}$ & +55 & ...... & $\ldots . .$. & $\ldots . .$. & .. & +177 & +193 \\
\hline
\end{tabular}

N.B. The numbers followed by a (d) are not strictly comparable with the others, as one of the substances is decomposed on boiling, so that its boiling-point cannot be determined with any degree of exactness.

The numbers marked with an * are exceptions to Relation 9. They are all due to the b.p. of propane, $\mathrm{C}_{3} \mathrm{H}_{8}$, being too high. $C f$, footnote to Tables $\mathrm{V}$. and VI. 
ReLation 9. (a) The differences referred to in Relation 8 increase algebraically as the algebraic difference between the atomic weights of the hydrocarbon radicals increases. (See Table VII.)

(b) In the case where several pairs of hydrocarbon radicals have a common difference in atomic weights, then the differences between the boiling-points of their halogen compounds increase as the sum of the atomic weights of the several pairs of hydrocarbon radicals increases. This rule also holds for the alkyl compounds so long as the difference between the boiling-points is a minus quantity ( $i$. e. so long as the boiling-point of the alkyl compound of the polyvalent hydrocarbon radical is lower than that of the corresponding alkyl compound of the monovalent hydrocarbon radical), but when this difference becomes a plus quantity the rule is reversed. (See Table VII.)

For the purpose of illustrating the above relationships wo have chiefly made use of the compounds of the sixth and seventh groups of hydrocarbon radicals, because the sets of compounds belonging to these groups are much more complete than in the case of the other groups ; the rules, however, hold equally with the latter as with the former.

The above nine rules have been applied in 1997 cases, of which 71 (or 3.6 per cent.) are exceptions. The exceptions are distributed as follows:-

\begin{tabular}{|c|c|c|c|c|c|c|c|c|}
\hline & \multicolumn{4}{|c|}{ Halogen Compounds. } & \multicolumn{4}{|c|}{ AlkyL Compounds. } \\
\hline & \multicolumn{2}{|c|}{$\begin{array}{l}\text { Melting- } \\
\text { points. }\end{array}$} & \multicolumn{2}{|c|}{$\begin{array}{l}\text { Boiling- } \\
\text { points. }\end{array}$} & \multicolumn{2}{|c|}{$\begin{array}{l}\text { Melting- } \\
\text { points. }\end{array}$} & \multicolumn{2}{|c|}{$\begin{array}{l}\text { Boiling- } \\
\text { points. }\end{array}$} \\
\hline & $\begin{array}{l}\text { No. of } \\
\text { cases. }\end{array}$ & $\begin{array}{c}\text { No. of } \\
\text { excep- } \\
\text { tions. }\end{array}$ & $\begin{array}{l}\text { No. of } \\
\text { cases, }\end{array}$ & $\begin{array}{l}\text { No. of } \\
\text { excep- } \\
\text { tions. }\end{array}$ & $\begin{array}{l}\text { No. of } \\
\text { cases. }\end{array}$ & $\begin{array}{l}\text { No. of } \\
\text { excep- } \\
\text { tions. }\end{array}$ & $\begin{array}{l}\text { No. of } \\
\text { cases. }\end{array}$ & $\begin{array}{l}\text { No. of } \\
\text { excep- } \\
\text { tions. }\end{array}$ \\
\hline 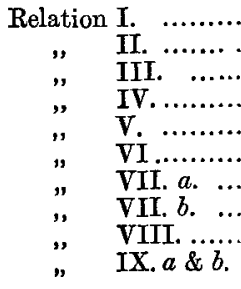 & $\begin{array}{l}20 \\
20 \\
\cdots \\
8 \\
1 \\
\cdots \\
\cdots \\
\cdots \\
\cdots\end{array}$ & $\begin{array}{c}2 \\
0 \\
\cdots \\
2 \\
0 \\
\cdots \\
\cdots \\
\cdots \\
\cdots \\
\cdots\end{array}$ & $\begin{array}{r}68 \\
60 \\
104 \\
35 \\
66 \\
78 \\
100 \\
103 \\
108 \\
106\end{array}$ & $\begin{array}{l}1 \\
1 \\
1 \\
1 \\
1 \\
0 \\
1 \\
0 \\
0 \\
0 \\
2\end{array}$ & $\begin{array}{c}5 \\
8 \\
6 \\
\cdots \\
\cdots \\
\cdots \\
\cdots \\
2 \\
\cdots \\
\cdots\end{array}$ & $\begin{array}{c}0 \\
0 \\
0 \\
\cdots \\
\cdots \\
\cdots \\
\cdots \\
0 \\
\cdots \\
\cdots\end{array}$ & $\begin{array}{r}48 \\
49 \\
73 \\
26 \\
30 \\
42 \\
73 \\
102 \\
285 \\
361\end{array}$ & $\begin{array}{r}0 \\
0 \\
0 \\
0 \\
1 \\
6 \\
7 \\
14 \\
0 \\
32\end{array}$ \\
\hline Total ............. & 49 & $=\begin{array}{c}4 \\
8 \%\end{array}$ & 828 & $\begin{aligned} & 7 \\
= & 0.8 \%\end{aligned}$ & 21 & 0 & 1089 & $\begin{array}{l}60 \\
=5.5 \%\end{array}$ \\
\hline
\end{tabular}


Of the above 71 exceptions no less than

45 are due to the b.p. of propane, $\mathrm{C}_{3} \mathrm{H}_{8}$, being too high.

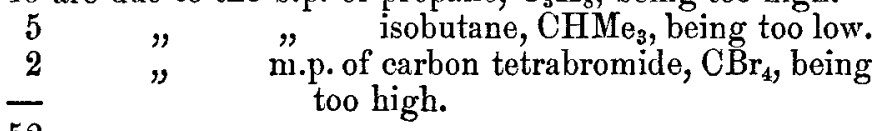

52

So that 52 of the 71 exceptions would be accounted for by errors in the boiling-points of two of the above compounds, and in the melting-point of the last one. As regards propane there is certainly a considerable error in its boiling-point, or rather in the number which has been assumed as the boilingpoint in our investigations ; for its actual boiling-point has not been determined, the value used being in fact the mean temperature of condensation, viz. $-25^{\circ}$ to $-30^{\circ}$ (cf. footnote to Table V.). With respect to isobutane, the boiling-point employed was that $\left(-17^{\circ}\right)$ given by Butlerow (Annalen, cxliv.10). Konovaloff states that it boils below $-10^{\circ}$ (Bull. Soc. Chim. (2) xxxiv. 333). Finally, carbon tetrabromide is not only an exception to some of the relations noted above, but it was also shown, in my paper on the Halogen Compounds of the Elements, that, owing to its abnormally high melting-point, it was the most marked exception to some of the rules there stated.

In conclusion it will be seen, by a comparison of this and my previous papers, that the above nine relationships, together with others of less importance, hold good for the following four classes of compounds :-

(1) The Halogen compounds of the Elements (i.e. of elements with elements).

(2) The Alkyl compounds of the Elements (i.e. of elements with hydrocarbon radicals).

(3) The Halogen compounds of the Hydrocarbon Radicals (i. e. of elements with hydrocarbon radicals).

(4) The Alkyl compounds of the Hydrocarbon Radicals (i.e. of hydrocarbon radicals with hydrocarbon radicals).

Though the periodicity of Relation 1 holds for all the four classes of compounds, yet the nature of the periodicity is not exactly the same throughout for the compounds of the hydrocarbon radicals as for those of the elements.

We are therefore justified in concluding that, with the exception just stated, the physical properties of the compounds of the bydrocarbon radicals (so far as investigated) follow the same rules and exhibit exactly the same relationships as the corresponding compounds of the elements.

In my next paper I hope to indicate the theoretical significance of this general fact. 
514 Prof. T. Carnelley on the Periodic Lav.

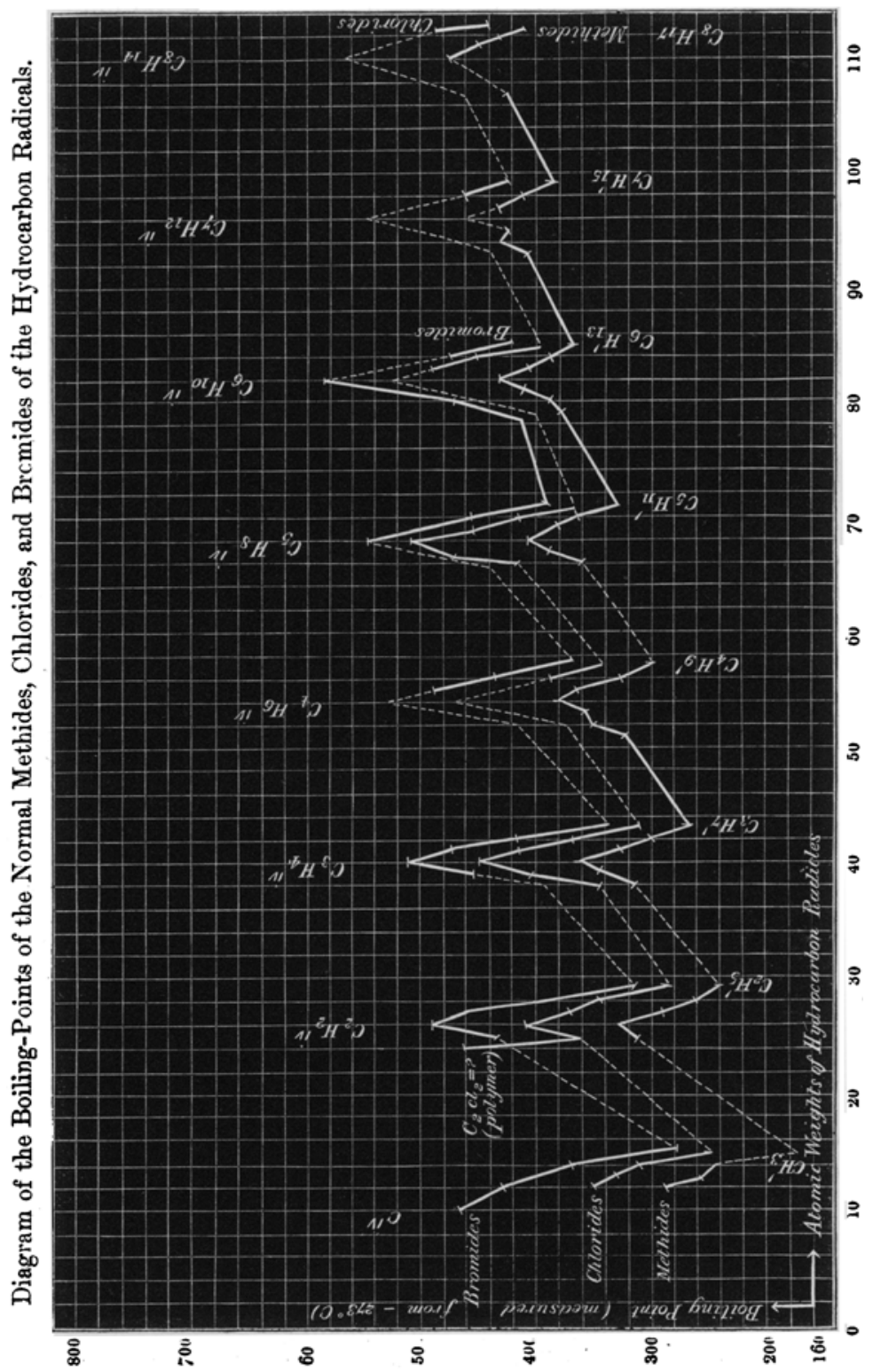

